\title{
The Effect of a Two-day Training and Refresher Program on the Basic Emergency Obstetric and Newborn Care Knowledge and Skills of Health Workers in Legazpi City, Albay
}

\author{
Ma. Stephanie Fay S. Cagayan, MD, PhD, ${ }^{1}$ Rita Mae Ang-Bon, MD, ${ }^{2}$ Fernando B. Garcia, Jr., MD, PhD, ${ }^{3}$ \\ Filomena S. San Juan, MD, PhD, ${ }^{1}$ Cecilia L. Llave, MD, PhD, ${ }^{1}$ \\ Catherine Banwell, $\mathrm{PhD}^{4}$ and Erlidia F. Llamas-Clark, MD, MPH, $\mathrm{PhD}^{1}$ \\ ${ }^{1}$ Department of Obstetrics and Gynecology, Philippine General Hospital, College of Medicine. University of the Philippines Manila \\ ${ }^{2}$ Department of Health Region $V$, Philippines \\ ${ }^{3}$ Department of Health Policy and Administration, College of Public Health, University of the Philippines Manila \\ ${ }^{4}$ Research School of Population Health, Australian National University
}

\begin{abstract}
Objectives. The availability of emergency obstetric and newborn care (EmONC) services is one of the key strategies in improving maternal health and achieving Sustainable Development Goal 3. Health staff knowledge and competency on these interventions are crucial to ensure the effective handling and management of obstetric and newborn emergencies. Health workers, despite having undergone formal training, require regular refresher courses to keep up with new, evidence-based information on EmONC intervention; and to ensure compliance with national and local referral guidelines. A two-day workshop was implemented to assess the knowledge and skills of health workers in Legazpi, Albay, regarding obstetric emergency cases and referral guidelines.
\end{abstract}

Methods. A pretest was conducted on the first day to assess the knowledge of the participants. A series of lectures were given before administering a posttest at the end of these lectures. An Objective Structured Clinical Examination (OSCE) was conducted on the second day, which aimed to assess the skills of the participants.

Results. The pretest findings indicated that the participants are generally knowledgeable about areas such as handwashing and prenatal care. Results of the posttest show that participants had difficulty with the category of referrals. Moreover, comparing the pretest and posttest scores, there is strong evidence that there is a difference in the median values of the pretest scores as compared to the posttest scores. The results of the OSCE also indicate the need for further training on partograph use and adherence to an EINC protocol. More than half of the participants passed all five parts of the OSCE.

Conclusion. The workshop contributed to an improvement in the knowledge of health care workers in obstetric emergency cases. Skills-wise, more frequent training may need to be conducted to improve other competencies such as partograph utilization and the practice of EINC interventions.

Key Words: emergency obstetric care, training, skilled birth attendants, knowledge, skills

\section{INTRODUCTION}

Corresponding author: Ma. Stephanie Fay S. Cagayan, MD, PhD Department of Pharmacology and Toxicology College of Medicine

University of the Philippines Manila

Salcedo Hall Building

547 Pedro Gil St., Ermita, Manila 1000, Philippines

Email: mscagayan@up.edu.ph
Several strategic developments have been done by the Philippine Department of Health (DOH) in the last five years as part of their commitment to contributing towards achievement of maternal and newborn survival-related Sustainable Development Goals (i.e., to reduce global MMR to less than 70 per 100,000 live births and NMR to at least as low as 12 per 1000 live births by 2030). ${ }^{1}$ One such 
strategy that has been promoted to reduce both maternal and newborn mortality is the timely access to comprehensive emergency obstetric and newborn care $(\mathrm{CEmONC})$ and the provision of at least the basic emergency obstetric and newborn care (BEmONC) at the primary health care level. ${ }^{2}$

Several studies have already demonstrated the effectiveness of EmONC interventions in reducing maternal mortality in low to middle-income countries. Some of these studies show that in resource-limited countries, programs integrating multiple interventions - including EmONC training, placement of skilled birth attendants, care providers, upgrading of facilities, and increase in the provision of essential drugs, supplies, and equipment - are likely to have a significant positive impact on maternal and newborn health..$^{3,4,5}$

Despite these developments, several studies have also shown that most health care facilities in developing regions of the world are still unable to provide emergency obstetric services. ${ }^{6,7}$ A systematic review done by Jonas et al. has shown that inadequate knowledge and skills among health providers hinder their capability to provide highquality EmONC. ${ }^{8}$ Another review conducted by Fikre also concluded that the inadequacy of essential equipment, shortages of health providers and the poor skills of available providers all account for the inadequate provision of EmONC in developing countries. ${ }^{9}$

A similar situation could be seen in the Philippines, particularly in its provinces. Several factors are affecting the slow decline of maternal and neonatal mortality rates in the country and one of these factors is the lack of skilled birth attendants who can provide high-quality EmONC and are also competent enough to make referrals of complicated cases to the provincial hospitals. Unfortunately, there is a severe maldistribution of trained and competent health workers in the private and public settings, particularly in obstetric care. ${ }^{10}$ This problem, like other developing countries, is further compounded by difficult geographical terrain, expensive transport costs, under-equipped healthcare facilities, and the congestion of patients within facilities. ${ }^{11,12}$

One major approach in solving this issue is to increase the provision of competency-based training and efforts which aim to promote the retention of practical knowledge and skills by health workers. ${ }^{13,14}$ Several studies also suggest that short competency based EmONC training programs and refresher courses are more effective in improving professional practice than longer didactic based training. ${ }^{15,16}$

Another strategy in maternal and neonatal mortality reduction is the strengthening of referral systems which, again, is highly reliant on the presence of competent and capable health workers. The Philippine government has already recognized the need for an effective referral system; as this is very much embedded in the Department of Health's (DOH) Maternal, Newborn and Child Health and Nutrition (MNCHN) strategy. ${ }^{17}$ The province of Albay in Bicol established the Interlocal Health Zone (ILHZ)
Service Delivery Network (SDN) guidelines in 2014 to further improve their maternal referral system and to decrease the province's maternal mortality ratio (MMR) as the Bicol region has consistently higher MMR than other provinces in the country. ${ }^{18,19}$

Training programs are conducted not only for capacitybuilding but as monitoring and evaluation tools to improve compliance with national and local referral guidelines. There is a need then for the continuous orientation, reorientation, and training of health care providers not only in providing EmONC but in ensuring compliance to guidelines for conducting referrals. Thus, this study is conducted to examine the effect of a two-day training and refresher program on the knowledge of high-risk obstetrics management, referral guidelines and EmONC skills of health workers in Legazpi City, Albay.

\section{MATERIALS AND METHODS}

\section{Study site and participants}

Legazpi City, Albay was selected as the study site based on the high maternal mortality rate in the Bicol region, presence of an interlocal health zone referral system, easy accessibility to referral services, availability of staff to provide obstetric services, presence of $\mathrm{DOH}$ accredited hospitals and the apex hospital of the provincial referral system in the city (Bicol Regional Training and Teaching Hospital), and most importantly, expressed willingness of the regional $\mathrm{DOH}$ and other stakeholders in the locality to participate in the study.

The target participants were all the local health workers involved in obstetric care, more specifically those barangays with high maternal mortality and morbidity in the last year. Those who refused to provide informed consent are excluded from participating in the study.

\section{SDN workshop and BEmONC refresher course}

A two-day training and refresher workshop as designed by the Philippine Obstetrical and Gynecological Society Committee on Maternal Mortality Reduction was implemented to assess and improve the knowledge and skills of health workers on obstetric emergency cases..$^{20}$ During the introduction to the workshop, a pretest was given to assess the knowledge of the participants. A series of lectures and skills demonstration was then conducted on the following topics: Maternal status in the Philippines, Respectful Maternity Care, Standard precaution and cleanliness, antenatal care, obstetric complications (e.g. hypertension in pregnancy, preterm labor), partograph interpretation, essential intrapartum and newborn care (EINC), postpartum hemorrhage and postnatal care, and LEDACAMARA (Legazpi, Daraga, Camalig, Manito and Rapu-rapu) SDN referral guidelines. A posttest was given at the end of the lectures. The second day of the workshop evaluated the skills of the health workers through the Objective Structured Clinical Examination (OSCE). The OSCE consisted of five 
stations: Partograph, EINC, magnesium sulfate (MgS04) injection with handwashing demonstration and postpartum hemorrhage. Participants were asked to perform the various skills required per station using the performance checklist adapted from the DOH BEmONC Module for Midwives, 2015. A passing grade of $60 \%$ was set per station.

\section{Analytical strategy}

Item analysis was done and data was then transferred into SPSS, and subjected to 2-way repeated ANOVA to determine any change from pretest to posttest scores. Linear regression analysis was also conducted to determine whether variables such as years in training, prior BEmONC training and place of work will affect scores.

\section{Ethical considerations}

The study received ethical clearance from the University of the Philippines Manila (UPM) Research Ethics Board as part of the bigger evaluation study done by the principal investigator (UPM REB 2018-010-01). The participants were informed of the purpose of the study, and consent was also obtained from them to conduct the study. They were assured of the confidentiality of the information obtained during the orientation before the workshop. No names or specific contact information were obtained from the participants. Participants were also given feedback regarding their performance during a City Health Office consultative meeting with health workers a month after. The $\mathrm{DOH}$ Regional Office was given an aggregate anonymized result of the performance of the participants.

\section{RESULTS}

\section{Description of the participants}

There were 22 health workers from public institutions and 18 health workers from private institutions who participated in the workshop. From the participants, $33(82.5 \%)$ are

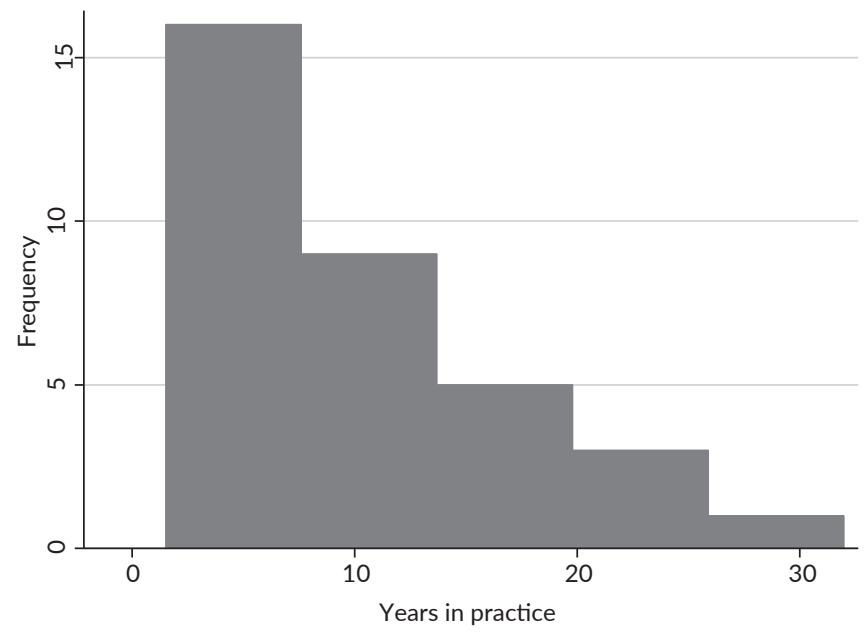

Figure 1. Histogram of the number of years in practice $(n=34)$. registered midwives, $3(7.5 \%)$ are registered nurses, 1 (2.5\%) is a physician, and $3(7.5 \%)$ are registered midwife and nurses.

The participants' years of service ranged from 1.5 years to 32 years, with a mean of $9.3 \pm 7.5$ years and a median of 9 years. The number of years in service is skewed to the right (Figure $1 ; p<0.01$ ).

Sixteen respondents $(40.0 \%)$ reported that they have received $\mathrm{BEmONC}$ training, while 18 respondents (45.0\%) reported that they have not received $\mathrm{BEmONC}$ training. Six (15.0\%) respondents did not report whether or not they have received $\mathrm{BEmONC}$ training.

\section{Knowledge assessment}

A pretest was done to assess the knowledge of the participants before the training. The pretest consisted of 25 multiple-choice items, and the areas evaluated during the written pretest were their knowledge on the following topics: handwashing, prenatal care, hypertension, EINC, use of partograph, referral guidelines and categories, preterm labor, postnatal care, and patient's rights. A total of 32 participants took the pretest, while 38 took the post text exam. The discrepancy is because six people who were present during the posttest were not present at the time that the pretest was administered (Table 1). Moreover, 2 participants attended but were not able to submit their pretest and posttest papers.

For the pretest, most participants appear to be knowledgeable on some areas of prenatal care, and most of them found difficulty in providing a correct answer in the area of hypertension. On the other hand, the results of the posttest also show that participants gave the most number of correct answers on some areas of prenatal care and hypertension, while questions about the referral category garnered the least number of correct answers.

Pre-test scores ranged from 3 to 17 , with a mean of $10.5 \pm 3.3$ and a median of 10 . This variable is normallydistributed (Figure 2; $p=0.78$ ).

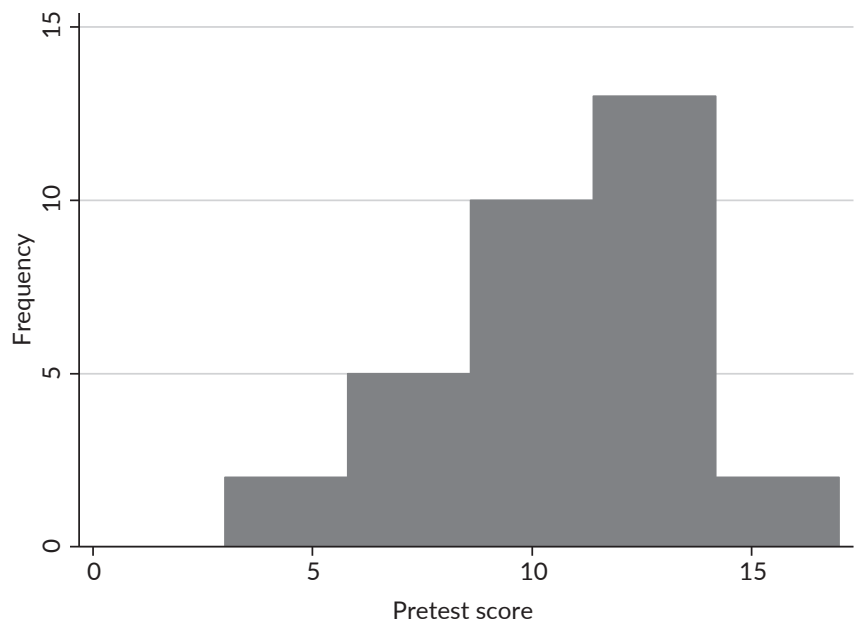

Figure 2. Histogram of pretest scores $(n=32)$. 
Table 1. Item analysis for the pretest and posttest

\begin{tabular}{|c|c|c|c|c|}
\hline \multirow{2}{*}{ Question } & \multicolumn{2}{|c|}{ Pretest $(n=32)$} & \multicolumn{2}{|c|}{ Posttest (n=38) } \\
\hline & Correct & Wrong & Correct & Wrong \\
\hline Q1 Infection Control & $14(43.8)$ & $18(56.3)$ & $18(47.4)$ & $20(52.6)$ \\
\hline Q2 ANC & $20(62.5)$ & $12(37.5)$ & $38(100.0)$ & $0(0.0)$ \\
\hline Q3 ANC & 7 (21.9) & $25(78.1)$ & $16(42.1)$ & 22 (57.9) \\
\hline Q4 ANC, Referral & $17(53.1)$ & $15(46.9)$ & $36(94.7)$ & $2(5.3)$ \\
\hline Q5 Hyperetension & $6(18.8)$ & $26(81.3)$ & 35 (92.1) & $3(7.9)$ \\
\hline Q6 EINC & $18(56.3)$ & $14(43.8)$ & $36(84.2)$ & $6(15.8)$ \\
\hline Q7 EINC & $13(40.6)$ & $19(59.4)$ & 32 (84.2) & $6(15.8)$ \\
\hline Q8 EINC & $15(46.9)$ & $17(53.1)$ & $30(79.0)$ & $8(21.1)$ \\
\hline Q9 Partograph & $16(50.0)$ & $16(50.0)$ & $37(97.4)$ & $1(2.6)$ \\
\hline Q10 Referral & $10(31.3)$ & $22(68.8)$ & 33 (86.8) & $5(13.2)$ \\
\hline Q11 Partograph & $12(37.5)$ & $20(62.5)$ & $33(86.8)$ & $5(13.2)$ \\
\hline Q12 EINC & $15(46.9)$ & $17(53.1)$ & 31 (81.6) & $7(18.4)$ \\
\hline Q13 Preterm Labor & $13(40.6)$ & $19(59.4)$ & $37(97.4)$ & $1(2.6)$ \\
\hline Q14 Postnatal Care & $8(25.0)$ & $24(75.0)$ & $21(55.3)$ & $17(44.7)$ \\
\hline Q15 Hypertension, Referral & $12(37.5)$ & $20(62.5)$ & 35 (92.1) & $3(7.9)$ \\
\hline Q16 Referral & $17(53.1)$ & $15(46.9)$ & $32(84.2)$ & $6(15.8)$ \\
\hline Q17 Hypertension & $12(37.5)$ & $20(62.5)$ & $38(100.0)$ & $0(0.0)$ \\
\hline Q18 Patient's Rights & $18(56.3)$ & $14(43.8)$ & 35 (92.1) & $3(7.9)$ \\
\hline Q19 Patient's Rights & $18(56.3)$ & $14(43.8)$ & $36(94.7)$ & $2(5.3)$ \\
\hline Q20 Patient's Rights & $18(56.3)$ & $14(43.8)$ & $36(94.7)$ & $2(5.3)$ \\
\hline Q21 Referral & $14(43.8)$ & $18(56.3)$ & $27(71.1)$ & $11(29.0)$ \\
\hline Q22 Referral & $16(50.0)$ & $16(50.0)$ & $34(89.5)$ & $4(10.5)$ \\
\hline Q23 Referral & $12(37.5)$ & $20(62.5)$ & $23(60.5)$ & $15(39.5)$ \\
\hline Q24 Referral & $8(25.0)$ & $24(75.0)$ & $14(36.8)$ & $24(63.2)$ \\
\hline Q25 Referral & $7(21.9)$ & $25(78.1)$ & $16(42.1)$ & $22(57.9)$ \\
\hline
\end{tabular}

Posttest scores ranged from 15 to 22, with a mean of $19.9 \pm 1.6$ and a median of 20 . This variable is left-skewed (Figure $3 ; p=0.03$ ).

The difference between posttest and pretest scores ranged from 3 to 17 , with a mean of $9.3 \pm 3.3$ and a median of 8. This variable is normally-distributed (Figure $4 ; p=0.38$ ).

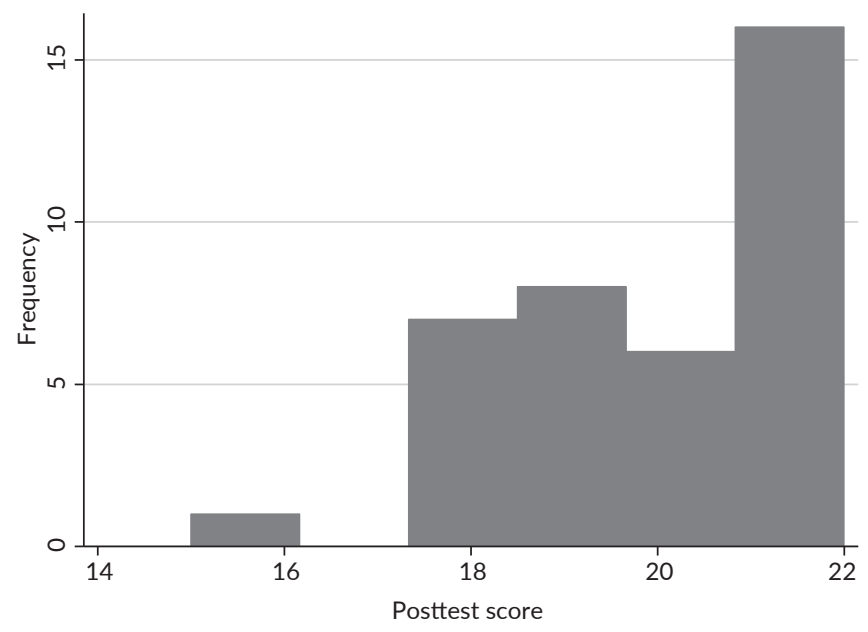

Figure 3. Histogram of posttest scores $(n=38)$.
As the posttest scores were left-skewed, the paired t-test cannot be used. As such, its non-parametric equivalent, the Wilcoxon signed-rank test, was used instead. Comparing the pretest scores with the posttest scores using the latter test, there is strong evidence that there is a difference in the median values of the pretest scores as compared to the posttest scores $(p<0.01)$.

\section{Skills test}

Correspondingly, the skills of the pretest participants were assessed through an OSCE. Thirty-two health workers participated in the OSCE. The examination covered the following areas: basic handwashing skills, EINC, administration of magnesium sulfate, using a partograph and management of postpartum hemorrhage.

\section{Handwashing skills}

Infection prevention measures such as handwashing is an essential skill for all health workers and are part of the routine care to be given for all mothers and babies. All 32 participants passed this station. The scores ranged from 6.6 to 11 , out of a maximum of 11 . The mean score is $10.5 \pm 0.9$, while the median score is 11 . The distribution of handwashing scores is left-skewed (Figure 5; $p<0.01$ ). 


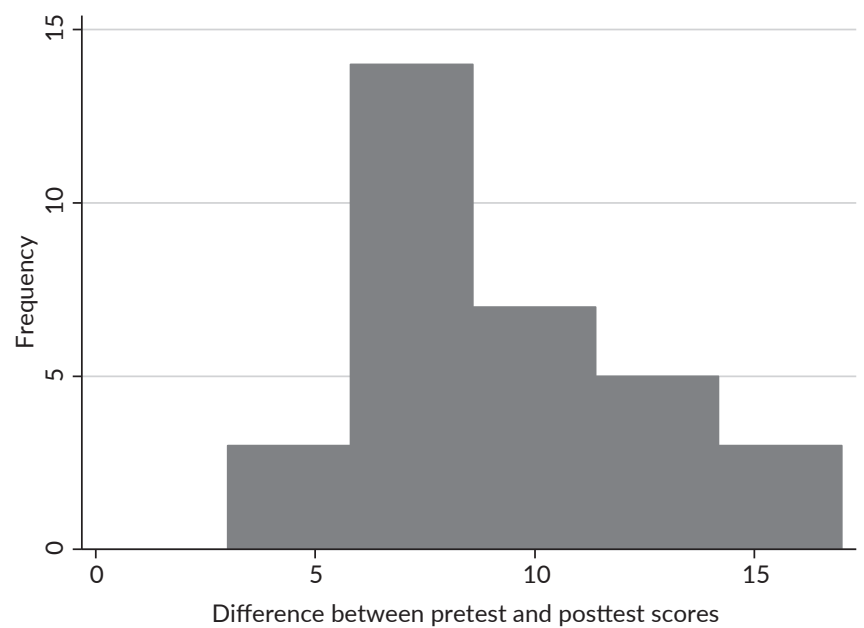

Figure 4. Histogram of the differences between pretest and posttest scores $(n=32)$.

\section{Essential Intrapartum and Newborn Care (EINC)}

The EINC practices are evidenced-based standards for safety and quality care of birthing mothers and their newborns, within the 48 hours of the intrapartum period and a week of life for the newborn. For this skill, $68.8 \%$ of the participants passed, while the remaining $31.3 \%$ failed. The scores ranged from 13.25 to 26.75 out of a maximum of 31 . The mean score is $20.3 \pm 3.6$, while the median score is 21.1 . The EINC scores are normally-distributed (Figure 6; $p=0.37$ ).

\section{Administration of Magnesium Sulfate}

The knowledge and skill in the administration of parenteral magnesium sulfate for the treatment of preeclampsia/eclampsia are essential in providing basic emergency care for hypertensive pregnant mothers. The results of the OSCE indicated that all participants passed this part of the test. The scores ranged from 5.4 to 9 , out of a

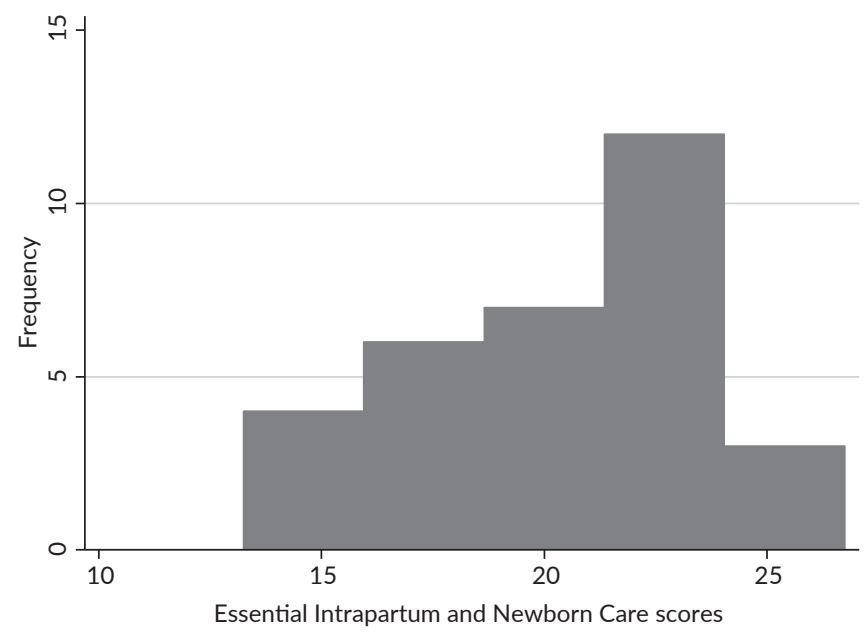

Figure 6. Histogram of Essential Intrapartum and Newborn Care scores $(n=32)$.

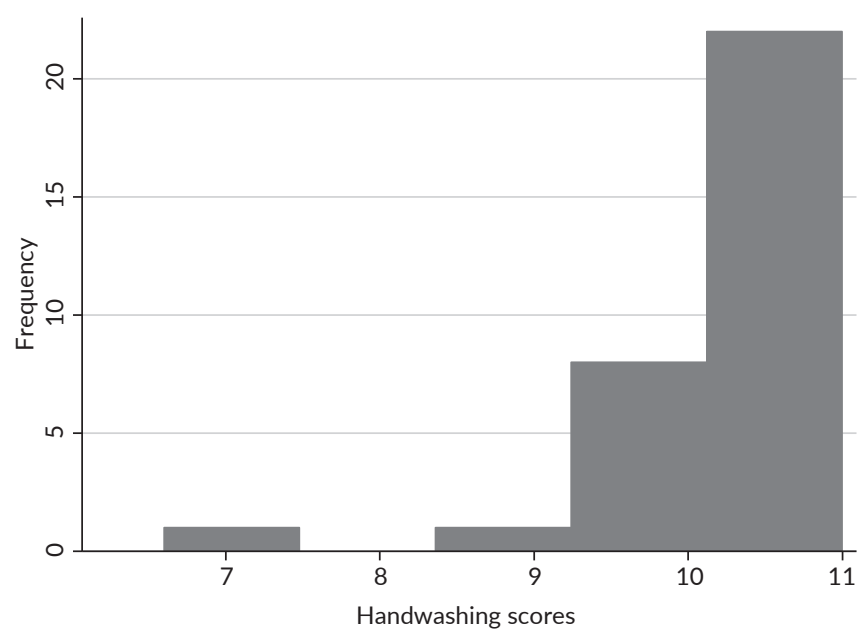

Figure 5. Histogram of handwashing scores $(n=32)$.

maximum of 9 . The mean score is $7.7 \pm 0.9$, while the median score is 8 . The distribution of scores for the administration of magnesium sulfate is left-skewed (Figure 7; $p=0.03$ ).

\section{Partograph use}

Health workers should be knowledgeable in monitoring and managing labor using the partograph for timely referral of obstructed labor to higher centers. For this skill, $87.5 \%$ of the participants passed, while $12.5 \%$ failed. The scores ranged from 5.25 to 13 , out of a maximum of 13 . The mean score is $11.4 \pm 2.1$, while the median score is 12 . The distribution of scores for using the partograph is left-skewed (Figure $8 ; p<0.01$ ).

\section{Postpartum Hemorrhage Management}

Postpartum hemorrhage is a life-threatening condition and all health workers providing obstetric care should know

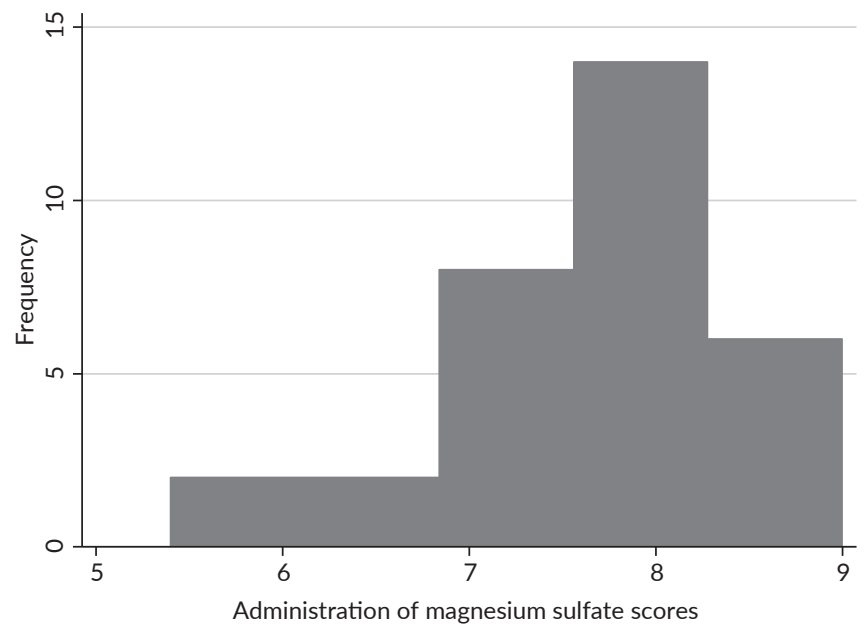

Figure 7. Histogram of administration of magnesium sulfate scores $(n=32)$. 
Table 2. The final model for test scores, receiving BEmONC training, working in public or private facility, and years in service

\begin{tabular}{lcccc}
\multicolumn{1}{c}{ Fixed-effects parameters } & Coefficient & Standard Error & $\boldsymbol{p}$-value & 95\% Confidence Interval \\
Years in practice & 0.06 & 0.14 & 0.67 & $-0.22-0.34$ \\
Has BEmONC training & 1.28 & 1.90 & 0.50 & $-2.45-5.02$ \\
Working in public facility & -0.05 & 1.52 & 0.97 & $-3.03-2.93$ \\
Constant & 13.25 & 3.35 & $<0.01$ & $\mathbf{6 . 6 9 - 1 9 . 8 2}$ \\
\multicolumn{1}{c}{ Random-effects parameters } & Estimate & Standard Error & $\mathbf{9 5 \%}$ Confidence Interval \\
Constant & $7.95 \times 10^{-21}$ & $4.00 \times 10^{-20}$ & $4.17 \times 10^{-25}-1.51 \times 10^{-16}$ \\
Residual & 29.61 & 5.86 & -43.66 \\
\hline
\end{tabular}

how to manage this complication. All the participants were able to pass this area. The scores ranged from 9.75 to 15 , out of a maximum of 15 . The mean score is $12.2 \pm 1.4$, while the median score is 12 . The scores for managing postpartum hemorrhage are normally-distributed (Figure 9; $p=0.77$ ).

In summary, more than half $(59.4 \%)$ of the participants in the OSCE assessment passed all five skill sets. On the other hand, $37.5 \%$ passed four out of five parts, and only one participant (3.1\%) passed three out of five parts.

Repeated measures mixed-effects linear regression was used to compare pretest and posttest scores with variables such as years in practice, previous $\mathrm{BEmONC}$ training, and type of health care facility in which the health worker is currently practicing. Analysis indicated that there is no interaction between receiving $\mathrm{BEmONC}$ training and working in a public or private facility $(p=0.82)$ (Table 2 ).

There is no strong evidence that pretest and posttest scores vary between years in service. The same can be said for the following variables: previous $\mathrm{BEmONC}$ training, and the type of health care facility in which the health worker is currently working in.

Furthermore, the association between years in practice, receiving $\mathrm{BEmONC}$ training, working in a private or public facility, pretest scores, posttest scores, and difference in scores, with passing all five OSCE parts were also studied (Table 3).

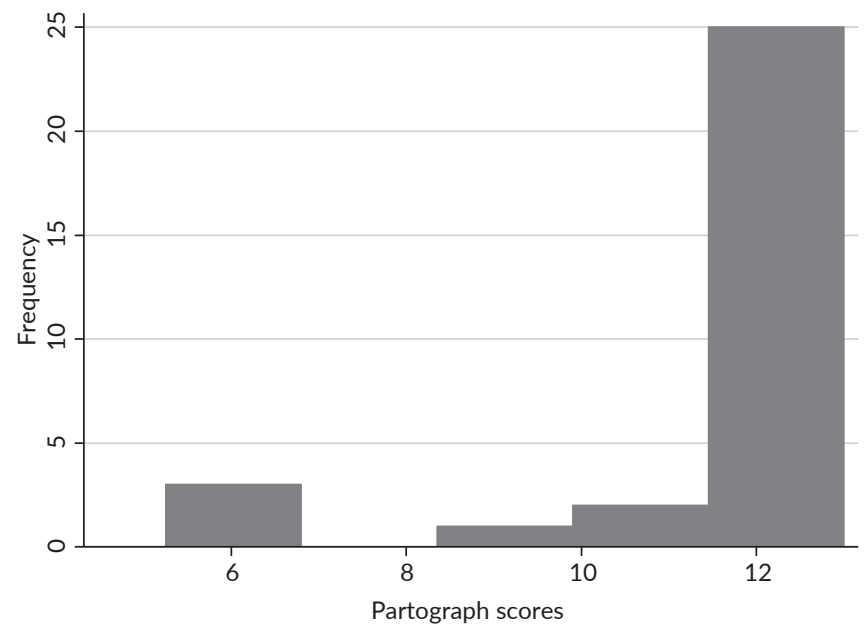

Figure 8. Histogram of partograph scores $(n=32)$.
Table 3. Cross-tabulations between receiving BEmONC training, working in private or public facilities

\begin{tabular}{lcrc} 
& \multicolumn{3}{c}{$p$-value } \\
\cline { 2 - 4 } & Not passed all & Passed all & $0.46^{*}$ \\
Workplace & & & \\
Private facility & $6(42.9)$ & $6(42.9)$ & \\
Public facility & $6(27.3)$ & $12(54.6)$ & \\
\hline BEmONC training & & & $0.71^{*}$ \\
No training & $6(33.3)$ & $8(44.4)$ & \\
Trained & $5(31.3)$ & $10(62.5)$ & \\
\hline
\end{tabular}

*Fisher's exact test

The proportion of those who passed all five OSCE aspects is higher among those working in public facilities as compared to those working in private facilities; and among those who have received $\mathrm{BEmONC}$ training as compared to those who do not. However, there is no strong evidence that these variables are associated with passing all OSCE aspects. There is no significant difference in the medians of the years in practice between those who passed all OSCE aspects, and between those who did not pass all OSCE aspects $(p=0.27)$. The mean pretest score for those who passed all OSCE parts is $10.1( \pm 3.5)$, while the mean pretest score for those who did not pass all OSCE parts is $10.9( \pm 2.7)$. There is no strong

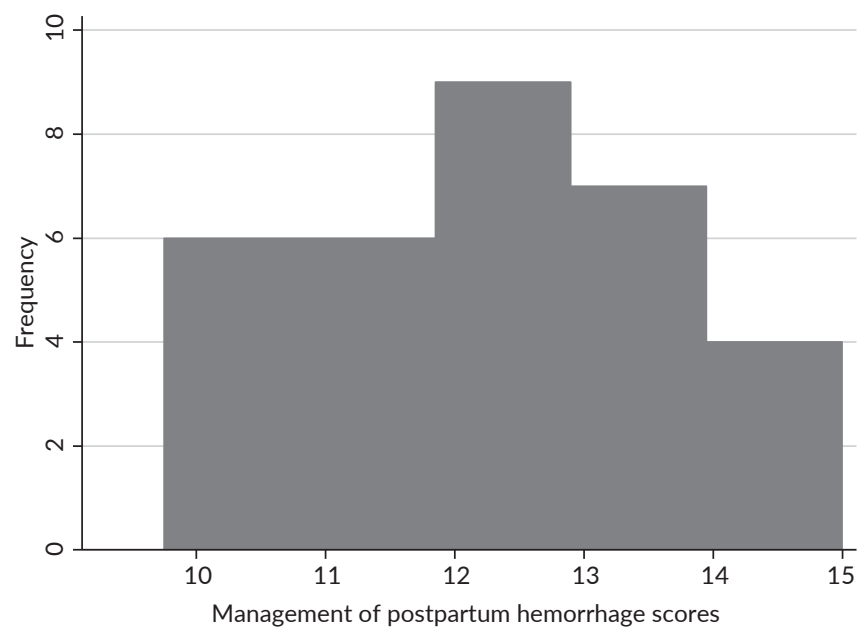

Figure 9. Histogram of management of postpartum hemorrhage scores $(n=32)$. 
evidence that the mean pretest score of those who passed all OSCE parts varied significantly from the mean pretest score of those who did not pass all OSCE parts $(p=0.54)$.

Moreover, there is no significant difference in the medians of the posttest scores between those who passed all OSCE aspects, and between those who did not pass all OSCE aspects $(p=0.59)$. There is also no strong evidence that the mean difference in the pretest and posttest scores for those who passed all OSCE parts varied significantly from the mean difference in the pretest and posttest scores of those who did not pass all OSCE parts $(p=0.61)$.

\section{DISCUSSION}

\section{Improvement in knowledge and skills}

Aside from the acquisition of knowledge, skills training is a vital component of any health care training program. Given the dynamic nature of medical care, frequent training programs and refresher courses are keys to enhancing knowledge and skills retention. This study was designed to investigate the effect of a two-day training and refresher program on the EmONC knowledge and skills of health providers in Legazpi City, Albay.

The pretest findings indicated that the participants were generally knowledgeable on the areas of handwashing and prenatal care. This could be attributed to their experience in their years of service as health workers specializing in obstetric and newborn care, previous BEmONC training, or previous opportunities for continuing professional education. Post-test results showed an improvement in the participants' knowledge in all the areas that were tested. However, of the knowledge areas tested, questions about their knowledge in conducting and assigning referrals had the least number of correct answers. Based on the results of the OSCE, on the other hand, participants demonstrated competency in handwashing, administration of parenteral magnesium sulfate and the management of postpartum hemorrhage. However, the OSCE findings also indicated that skills related to the use of the partograph and EINC interventions were lacking among most of the participants.

An obstetric referral is considered an important component of emergency care, necessary for reducing maternal mortality. The inability to refer an expectant mother in a timely fashion may lead to the development of lifethreatening complications, often resulting in preventable maternal deaths. Effective referral systems ensure that there is a continuum of care, as patients are referred from health centers in communities to a secondary level of care. ${ }^{21}$ The efficiency of an effective referral system may, however, be affected by factors such as lack of accessible transportation, socio-economic disparities, and inadequate training on the established referral guidelines. ${ }^{22,23}$

Based on the results of the OSCE, participants demonstrated competency in the skills of handwashing, administration of parenteral magnesium sulfate and the management of postpartum hemorrhage. However, OSCE findings also indicate that skills involving the use of partograph, and EINC interventions were lacking among most of the participants.

As for labor dysfunctions, the partograph is recommended by the World Health Organization (WHO) to monitor and record maternal and fetal well-being as it can be used to identify maternal or fetal distress and abnormalities in the progress of labor - factors which require immediate action, including a referral to a higher-level facility. ${ }^{24}$ One of the core skills of a trained birth attendant is the appropriate utilization of a partograph. Despite the benefits of this tool, challenges remain in the adoption of this labor tracker in lowto middle-income countries. ${ }^{25}$ Some of the factors which contribute to low partograph utilization are the following: lack of skilled and trained personnel, inadequate exposure or unavailability of the partographs, and absence of training on its use, and a lack of support and guidance..$^{26,27,28}$

Aside from the utilization of the partograph, another skill that needed reiterating among the participants was the practice of EINC. The EINC distinguishes the necessary practices in the delivery and care for the newborn and the mother. A study done in Iligan City examining the barriers to the implementation of the EINC protocol in public and private hospitals found that it was not done due to lack of funding, lack of space and facilities, and the lack of support from the administration to conduct training on the EINC protocol. The lack of training and seminars had a significant relationship with the EINC protocol's implementation. It is recommended that constant training and orientation of new doctors, nurses and trainees is crucial to ensure the implementation of the EINC protocol. ${ }^{29}$

\section{Global framework on evaluating EmONC training}

Several studies have shown that healthcare workers still lack the competency to provide all EmONC signal functions. ${ }^{30,31}$ The combination of inadequate knowledge and skills remains a primary reason as to why many beneficial evidence-based practices are still not in place. Health care providers should receive regular training to ensure their continued accreditation. Moreover, low and middle-income countries have often developed EmONC training programs for their healthcare providers as a significant component of their workplan and budgets. ${ }^{32}$

In this study, no difference in knowledge and skills results was seen between those who had prior BEmONC training and those who did not. It is crucial to determine the effectiveness of in-service EmONC training to make continuous improvements to the program, provide evidence to sustain the program and ensure that limited resources are well spent. The most commonly used training evaluation framework is that of Kirkpatrick's assessment of the effectiveness of adult education in combination with the internationally agreed indicators for availability and quality of EmONC. 33,34 
Kirkpatrick's framework recommends that a training program is evaluated in ten steps, which can be summarized in four levels. The first level determines the acceptability of the program. A positive reaction to training is indicative of interest which is a prerequisite to maximum learning. The second level evaluates the knowledge and skills acquired as a result of training. The third level determines the change in behavior after the training. Lastly, the fourth level aims to determine the tangible costs of the training in terms of reduced cost, improved quality, improved customer satisfaction, and improved productivity.

Using the global recommendation of Kirkpatrick's framework, it is revealed that in this study, level 1 evaluation has not been carried out. No evidence shows the acceptability of the program and how workers find it relevant to their day to day work and clinical settings. However, the result of the study provides evidence that there were significant improvements in knowledge and skills in the health care workers who attended the workshop and received training (Level 2). Level 3 evaluation of the workshop suggests that a behavioral change may not have been obtained due to the poor adherence to the EINC protocol. Lastly, there is insufficient data to suggest that the workshop resulted in improved health outcomes (Level 4 evaluation).

It can be recommended that the SDN workshop and refresher course should be implemented using evidence-based approaches while also ensuring routine but a comprehensive evaluation of effectiveness. The use of indicators and tools to measure change in practice and change in health outcomes after training may be utilized. Moreover, the long-term impact of the workshop on the management of obstetric complications and maternal and newborn mortality and morbidity should also be assessed. Lastly, the provision of opportunities to practice skills to promote long term retention of knowledge and skills obtained after training should also be done.

\section{Recommendations on scaling}

Scaling up the BEmONC training program entails reaching a larger number of people in a potentially broader geographical area. Several studies recommend the use of stages of change framework in setting the implementation and scale-up agenda. ${ }^{35,36}$ The framework consists of three phases. In the pre-implementation phase, two stages are distinguished: (1) creating awareness of the problem and acknowledging that something must be done; (2) committing to implement and scale up the EmONC program to a facility level, district level, provincial level or national level. This entails policy support and strategic planning from healthcare users, providers, and policymakers.

The implementation phase also consists of two stages namely 1) implementation of the training program which includes documentation of improvements (e.g. service, referral, morbidity and mortality improvements) and 2) the integration of the training program into routine practice.
Interventions for this stage include regular feedback, and continued refresher training and emergency drills.

The last phase is the institutionalization phase which consists of integrating the program or intervention into routine practice and sustaining the new practice. This entails long term and sustained monitoring, evaluation, and feedback on scale-up and the provision of the EmONC services.

Despite the recommendation of this framework, the conditions required for scaling up remain complex and include the following: Awareness and engagement from political and administrative bodies, creating an engaging policy environment leading to commitment, health systems strengthening, obtaining resources (financial and human resources for health), dissemination and training, continuous supervisory support, and utilizing an appropriate monitoring and evaluation approach. Scaling up the $\mathrm{BEmONC}$ training program may be recognized as a critical step in addressing the burden of maternal morbidity and mortality in Legazpi, Albay. Implementation, scale-up, and sustainability are not linear processes but require multi-sector involvement thus lending itself into a program that is not only successfully implemented but can also be sustained.

\section{Study Limitations}

Despite the invitation of the $\mathrm{DOH}$ regional office to all health care workers involved in obstetric care in Legazpi City to attend the workshop, only 40 health workers attended and the majority of them were non-doctors. This small number of participants and unequal distribution of birth attendants may have skewed the results of this study. It is therefore recommended that even before scaling up of this program is done, this study be validated on a larger number of health professionals with equal distribution of doctors, nurses, and midwives in other local government units with different rates of maternal and neonatal mortality and morbidity to gain a better perspective of the relationship of levels of knowledge and skills to management of emergency obstetric cases.

\section{CONCLUSION}

Obstetric and neonatal emergencies are sometimes inevitable. Therefore, staff competency in managing complications is paramount. The utilization of skilled birth attendants is deemed crucial in preventing maternal morbidity and mortality. In pursuit of this global commitment to reduce maternal deaths, the $\mathrm{DOH}$ in partnership with local government units is continuously implementing health reforms and programs geared towards the MNCHN strategy. The strategy highlights the importance of having committed skilled health professionals able to provide quality health interventions that are responsive to the needs of women and newborns. In line with this strategy, a two-day workshop was developed and consequently was found to be beneficial in improving the knowledge and skills of health workers in providing BEmONC. However, the suboptimal 
performance of some skills such as the use of the partograph, and adherence to EINC protocol, may indicate the need for ongoing refresher seminars and training on these specific skills. It is recommended that comprehensive monitoring and evaluation frameworks are utilized to evaluate the effectiveness of the training program. Moreover, preparation for scaling up begins with committed leaders, human resource capacity, competency building, and continuous monitoring and evaluation.

\section{Acknowledgments}

The authors would wish to acknowledge the support of the health staff of the different facilities in Legazpi City, Albay, as well as the research assistants during the study. We would also like to express our sincerest gratitude to the editors and referees for their valuable inputs and comments on this manuscript.

\section{Statement of Authorship}

All authors participated in research conceptualization and data interpretation and analysis, critical revision of article and final approval of version to be published.

\section{Author Disclosure}

All authors declared no conflicts of interest.

\section{Funding Source:}

This paper was funded by UP-NIH with grant \# NIH 2018-001.

\section{REFERENCES}

1. Dayrit MH. [Internet]. [cited 2019 April 27]. Philippines' struggles to lower maternal mortality. 2015. Available from: https://www. scidev.net/asia-pacific/health/opinion/philippines-struggles-to-lowermaternal-mortality.html

2. WHO, UNICEF, UNFPA, AMDD. Monitoring emergency obstetric care: a handbook. Geneva: World Health Organization; 2009.

3. Paxton A, Maine D, Freedman L, Fry D, Lobis S. The evidence for emergency obstetric care. Int J Gynecol Obstet. 2005;88(2):181-93.

4. Lindtjorn B, Mitiku D, Zidda Z, Yaya Y. Reducing maternal deaths in Ethiopia: results of an intervention Programme in Southwest Ethiopia. PLoS One. 2017 Jan;12(1):e0169304.

5. Nyamtema AS, Urassa DP, van Roosmalen J. Maternal health interventions in resource limited countries: a systematic review of packages, impacts and factors for change. BMC Pregnancy Childbirth. 2011 Dec;11(1):30.

6. Ameh C, Adegoke A, Hofman J, Ismail FM, Ahmed, FM, van den Broek, N. The impact of emergency obstetric care training in Somaliland, Somalia. Int J Gynecol Obstet. 2012 Jun;117(3):283-7.

7. Echoka E, Kombe Y, Dubourg D, Makokha A, Evjen-Olsen B, Mwangi M, Byskov J, Olsen ØE, Mutisya R. Existence and functionality of emergency obstetric care services at district level in Kenya: theoretical coverage versus reality. BMC Health Serv Res. 2013 Dec;13(1):113.

8. Jonas K, Crutzen R, van den Borne B, Reddy P. Healthcare workers' behaviors and personal determinants associated with providing adequate sexual and reproductive healthcare services in sub-Saharan Africa: a systematic review. BMC Pregnancy Childbirth. 2017 Dec;17(1):86.

9. Fikre R. Healthcare provider and the quality of emergency obstetric care in health Centre level in developing countries: a systematic review of the literature. Journal of Health, Medicine and Nursing. 2016;25,85-90.

10. Cabral EI. The Philippine health agenda for 2016 to 2022. Philipp J Intern Med. 2016;54(2).

11. Mehata S, Paudel YR, Dariang M, Aryal KK, Lal BK, Khanal MN, Thomas D. Trends and inequalities in use of maternal health care services in Nepal: strategy in the search for improvements. Biomed Res Int. 2017;2017.

12. Wilunda C, Tanaka S, Putoto G, Tsegaye A, Kawakami K. Evaluation of a maternal health care project in South West Shoa Zone, Ethiopia: before-and-after comparison. Reprod Health. 2016 Dec;13(1):95.

13. Bongban NG, Meyer DJ, Gatunsi PM, Isabella M. Emergency obstetrics knowledge and practical skills retention among hospitals and clinic staff following advanced life support obstetrical training in the Cameroon, Africa. Front Womens Health. 2016;1.

14. Ameh CA, Kerr R, Madaj B, Mdegela M, Kana T, Jones S, Lambert J, Dickinson F, White S, van den Broek N. Knowledge and skills of healthcare providers in sub-Saharan Africa and Asia before and after competency-based training in emergency obstetric and early newborn care. PloS One. 2016 Dec;11(12):e0167270.

15. Van Lonkhuijzen L, Dijkman A, Van Roosmalen J, Zeeman G, Scherpbier A. A systematic review of the effectiveness of training in emergency obstetric care in low-resource environments, BJOG. 2010 Jun;117(7):777-787.

16. Forsetlund,L, Bjørndal A, Rashidian A, Jamtvedt G, O'Brien MA, Wolf F, Davis D, Odgaard-Jensen J, Oxman AD. Continuing education meetings and workshops: Effects on professional practice and health care outcomes. Cochrane Database Syst Rev. 2009;(15)2.

17. Department of Health. MNCHN Strategy Manual of Operations [Internet]. 2nd ed. Manila: Department of Health \& National Center for Disease Prevention and Control; 2011 [cited 2019 May 12]. Available from: https://www.doh.gov.ph/sites/default/files/ publications/MNCHNMOPMay4withECJ.pdf

18. Quintos M. Regional Differences in Maternal Mortality in the Philippines. Asia Pacific Journal of Education, Arts and Sciences. 2017;4(1):1-14.

19. FP-MNCHN Referral Guidelines for LEDACAMARA (Legazpi, Daraga, Camalig, Manito, Rapu-rapu) Service Delivery Network.

20. San Juan, FS, Llamas-Clark EF and Fernandez-Tan CS. Improving Maternal and Neonatal Care: a facilitator's training guide book. Adapted Basic Emergency Obstetric and Newborn Care (BEmONC) Modules. Quezon City, Philippines. Philippine Obstetrical and Gynecological Society; 2018.

21. Ototorin E, Gomez P, Currie S, Thapa K, Dao B. Essential Basic and Emergency Obstetric and Newborn Care: From education and training to service delivery and quality of care. Int J Gynecol Obstet. 2015 Jun;130:S46-53.

22. WHO Western Pacific Region. [Internet]. 2019. [cited 2019 May 12]. Essential intrapartum and newborn care (EINC). Available from: http://www.wpro.who.int/philippines/areas/maternal_child_ nutrition/newborn_mother_care/en/

23. Macintyre K, Hotchkiss DR. Referral revisited: community financing schemes and emergency transport in rural Africa. Soc Sci Med. 1999 Dec;49(11):1473-87.

24. Shinde K, Bangal V, Singh R. Study of course of labour by using the modified WHO Partogram. Int J Biomed Adv Res. 2012;3:5.

25. Kigenyi O, Tefera GB, Nabiwemba E, Orach CG. Quality of intrapartum care at Mulago national referral hospital, Uganda: clients' perspective. BMC Pregnancy Childbirth. 2013 Dec;13(1):162.

26. Opiah MM, Ofi AB, Essien EJ, Monjok E. Knowledge and utilization of the partograph among midwives in the Niger Delta region of Nigeria. Afr J Reprod Health. 2012 Mar;16(1):125-32.

27. Kayiga H, Ajeani J, Kiondo P, Kaye DK. Improving the quality of obstetric care for women with obstructed labour in the national referral hospital in Uganda: lessons learnt from criteria based audit. BMC Pregnancy Childbirth. 2016 Dec;16(1):152.

28. Ogwang S, Karyabakabo Z. Assessment of partogram use during labour in Rujumbura Health Sub District, Rukungiri district, Uganda. Afr Health Sci. 2009;9(S1):27-34. 
29. Bangcola AA, Caorong LA. Barriers to the implementation of essential intrapartum and newborn care protocol (EINC) in public and private hospitals in Iligan City. Glob J Med Res. 2016 Jun;16(3).

30. Adegoke AA, Van Den Broek N. Skilled birth attendance-lessons learnt. BJOG. 2009 Oct;116:33-40.

31. Utz B, Siddiqui G, Adegoke A. \& Van Den Broek, N. 2013. Definitions and roles of a skilled birth attendant: a mapping exercise from four South-Asian countries. Acta Obstet Gynecol Scand Suppl. 2013 Sep;92(9):1063-9.

32. Nyamtema AS, Urassa DP, van Roosmalen J. 2011. Maternal health interventions in resource limited countries: a systematic review of packages, impacts and factors for change. BMC Pregnancy Childbirth. 2011 Dec;11(1): 94-102.
33. Kirkpatrick DL, Kirkpatrick JD. 2007. Implementing the Four Levels a Practical Guide for Effective Evaluation of Training Programs. San Francisco: Berrett-Koehler Publishers.

34. World Health Organization, UNFPA, UNICEF \& AMDD. 2009. Monitoring Emergency Obstetric Care: A Handbook. Geneva: World Health Organization; http://whqlibdoc.who.int/ publications/2009/9789241547734_eng.pdf.

35. Belizán M, Bergh A-M, Cilliers C. 2011. Stages of change: A qualitative study on the implementation of a perinatal audit programme in South Africa. BMC Health Serv Res. 2011 Dec;11(1):243.

36. Bergh AM, Allanson E, Pattinson RC. 2015. What is needed for taking emergency obstetric and neonatal programmes to scale? Best Pract Res Clin Obstet Gynaecol. 2015 Nov;29(8):1017-27.

The Acta Medica Philippina is now accepting original scientific papers, review articles and case reports for its upcoming issues. Please follow the format for submission as indicated in the "Instructions to Authors" elsewhere in the journal. All papers received shall be properly acknowledged. For inquiries and submission of proposals, please email us at actamedicaphilippina.upm@up.edu.ph 